Check for updates

Cite this: Nanoscale Adv., 2019, 1, 1791

\title{
Tetradic phosphor white light with variable CCT and superlative CRI through organolead halide perovskite nanocrystals
}

\author{
Gopi C. Adhikari, (D) Preston A. Vargas, (DD Hongyang Zhu, Alexei Grigoriev \\ and Peifen Zhu (DD *
}

In this work, the emission spectral range of halide perovskite nanocrystals is extended from violet to infrared, the widest emission range for halide perovskites to date. This range extension was made possible by a cost-effective solution-based synthesis process that only involves two halides $\left[\mathrm{MAPb}\left(\mathrm{Br}_{x} \mathrm{I}_{1-x}\right)_{3}\right.$ and $\left.\mathrm{MA}=\mathrm{CH}_{3} \mathrm{NH}_{3}\right]$. Furthermore, the correlated-color temperature (CCT) of white light is tuned by blending an appropriate fraction of the as-synthesized blue, green, yellow, and red emitting nanocrystals. This represents one of the first applications of a tetradic phosphor system for maximizing the color rendering index (CRI) for this material. The CCT ranges from warm to cool white (2759-6398 $\mathrm{K})$ and the CRI has a maximum value of 93.95 . Thus, this fourfold phosphor approach demonstrates that halide perovskites are promising alternatives to conventional phosphors in the search for low-cost and high-quality white light sources in the next generation of white lighting technology.

Received 26th February 2019

Accepted 28th February 2019

DOI: 10.1039/c9na00125e

rsc.li/nanoscale-advances

attest to their future applicability in solid-state devices and may lead to rapid improvement in the performance of optoelectronic devices such as LEDs, ${ }^{34-38}$ solar cells, ${ }^{39-42}$ photodetectors, ${ }^{43,44}$ and lasers. ${ }^{45,46}$ Many publications have reported the fabrication of white light LEDs using halide perovskites. Some used perovskites as one of the visible color emitting materials combined with a UV/blue LED chip and conventional phosphors. ${ }^{23,47,48}$ Others have replaced conventional phosphors altogether, using only perovskites with various emission spectra [red, green, and blue (RGB)]. ${ }^{49-51}$ Recently, there has also been success in transforming halide perovskites into single-component whitelight emitters. ${ }^{52-54}$ Thus, there is great potential for nanocrystal integration with III-nitride UV/blue LEDs to achieve high-quality white light and colorful LEDs via the direct combination of materials with different emission spectra.

Here, we demonstrate a breakthrough in photon downconversion based white LED technology via halide perovskite phosphors. Methylammonium lead mixed-halide $\operatorname{MAPb}\left(\mathrm{Br}_{x^{-}}\right.$ $\left.\mathrm{I}_{1-x}\right)_{3}$ perovskite nanocrystals have been synthesized via a solution-based synthesis with stringent ligand-mediation and anion ratio control, resulting in a wide range of narrow line-width emission spectra. The photoluminescence (PL) emission ranges from $408 \mathrm{~nm}$ to $755 \mathrm{~nm}$ and the band gap extends from $3.04 \mathrm{eV}$ to $1.51 \mathrm{eV}$. Furthermore, we have estimated white light emission using the perovskite nanocrystals with four-band emission (blue, green, yellow, and red) instead of the usual three-band emission. ${ }^{26,49}$ By optimizing the contribution of each of the four components, we obtained tunable CCT ranging from 2759 to $6398 \mathrm{~K}$ and the CRI reached an unprecedented value of 93.95 . 


\section{Experimental section}

\section{Materials and methods}

The synthesis of $\mathrm{MAPbBr}_{3}$ began by adding $0.025 \mathrm{~g}$ of $\mathrm{MABr}$ and $0.05 \mathrm{~g}$ of $\mathrm{PbBr}_{2}$ to a mixture of $0.5 \mathrm{~mL}$ of dimethylformamide, 8.5 of $\mathrm{mL}$ gamma-butyrolactone, and $15 \mu \mathrm{L}$ of octylamine (consider, $15 \mu \mathrm{L}$ of the ligand octylamine as ' $L$ '). This mixture was stirred at $70-80{ }^{\circ} \mathrm{C}$ on a hot plate until the solids dissolved completely and the solution became transparent. Then, this precursor solution was slowly mixed with $5 \mathrm{~mL}$ of toluene under magnetic stirring at room temperature. The solution was then centrifuged at $5000 \mathrm{rpm}$ for $5 \mathrm{~min}$ to separate the aggregate particles. The supernatant was poured into a clean vial and centrifuged again at $16000 \mathrm{rpm}$ for $15 \mathrm{~min}$. The precipitate was then collected by discarding the supernatant. The $\mathrm{MAPbI}_{3}$ precursor solution was prepared using the same procedure as the $\mathrm{MAPbBr}_{3}$ precursor, using $0.036 \mathrm{~g}$ of MAI and $0.069 \mathrm{~g}$ of $\mathrm{PbI}_{2}$ to replace the bromine components. The $\mathrm{MAPbI}_{3}$ precursor was blended with the $\mathrm{MAPbBr}_{3}$ precursor in the ratio required to achieve the intended composition of $\operatorname{MAPb}\left(\mathrm{Br}_{x} \mathrm{I}_{1-x}\right)_{3}$. In order to shift the PL emission from green to violet, the ligand (octylamine) concentration was increased in the $\mathrm{MAPbBr}_{3}$ precursor only. After synthesis, the nanocrystals were dispersed in toluene for storage and further characterization. This synthesis was performed in an ambient atmosphere.

\section{Characterization}

X-ray diffraction (XRD) measurements were taken using a Rigaku Smart Lab system with a $\mathrm{Cu} \mathrm{K}_{\alpha 1}(\lambda=1.54 \AA)$ fixed anode X-ray source operating at $40 \mathrm{kV}$ and $44 \mathrm{~mA}$. Nanocrystal samples were placed on a glass substrate and dried in an ambient environment before XRD characterization.
Transmission electron microscopy (TEM) measurements were performed using a Hitachi H-7000 TEM at $75 \mathrm{kV}$. Nanocrystal samples for TEM measurements were prepared by diluting them in toluene and sonicating to separate the individual particles. The sample was then carefully placed onto a copper grid with a carbon film and dried in an ambient atmosphere before characterization. The UV-visible absorption spectra were measured using a VARIAN Carry 50 Scan UV-Spectrophotometer with nanocrystals in a toluene colloidal suspension. The PL spectra were measured using a Spectrofluorophotometer (Shimadzu, RF-6000) with a monochromatic xenon lamp as the excitation source while the nanocrystals remained in a colloidal suspension. The LED spectra were obtained by combining four different emissions with different ratios. The calculation of CRI and CCT were performed according to equations defined by the CIE (Commission Internationale de l'Eclairage). Four different emission spectra (blue, green, yellow, and red) were combined and the spectral data were analysed to yield the CRI, CCT, and CIE coordinates.

\section{Results and discussion}

The effects of ligand concentration and compositions of anions on the crystal structure were studied using XRD patterns (Fig. 1a). The sharp diffraction peaks confirm the high crystallinity of the nanocrystals. For $\mathrm{MAPbBr}_{3}$, the diffraction patterns are consistent with the cubic crystal structure with a $P m \overline{3} m$ (no. 221) space group. For $\mathrm{MAPbI}_{3}$, however, the diffraction scan shows a tetragonal crystal structure with an $I 4 / \mathrm{mcm}$ (no. 140) space group. ${ }^{55,56}$ No changes in the peak position of ligandmediated $\mathrm{MAPbBr}_{3}$ nanocrystals was observed from the XRD patterns. This indicates that the adjustment of the ligand concentration in the reaction environment has no effect on the

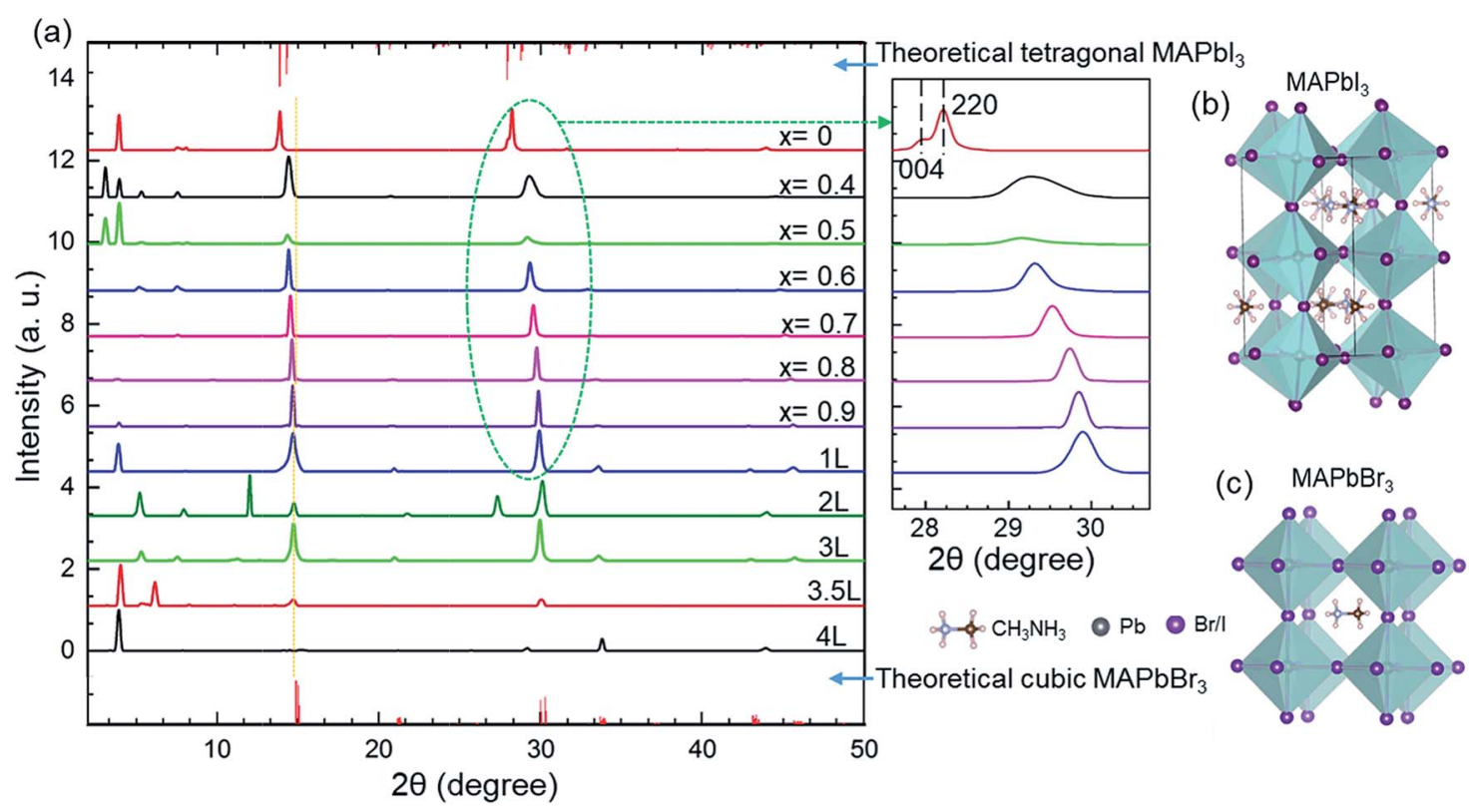

Fig. 1 (a) The XRD spectra of $\mathrm{MAPbBr}_{3}$ with varying ligand concentration and $\mathrm{MAPb}\left(\mathrm{Br}_{x} \mathrm{I}_{1-x}\right)_{3}$ with varying 'Br' : 'l' ratio. (b) The primitive cell of cubic-structured $\mathrm{MAPbBr}_{3}$. (c) The primitive cell of tetragonal-structured MAPbl $3 . L=15 \mu \mathrm{L}$ of ligand (octylamine). 
Table 1 Peak emission wavelengths, FWHM, and band gaps of $M \mathrm{MPbBr}_{3}$ with variable ligand amount $(L)$ as defined in the Experimental section

\begin{tabular}{llll}
\hline $\begin{array}{l}\text { Multiple of } \\
\text { ligand } \\
\begin{array}{l}\text { concentration } \\
(L)\end{array}\end{array}$ & $\begin{array}{l}\text { Peak emission } \\
\text { wavelength } \\
(\mathrm{nm})\end{array}$ & $\begin{array}{l}\text { FWHM } \\
(\mathrm{nm})\end{array}$ & $\begin{array}{l}\text { Band } \\
\text { gap (eV) }\end{array}$ \\
\hline 1 & 520 & 21 & 2.36 \\
2 & 510 & 25 & 2.52 \\
3 & 478 & 26 & 2.73 \\
3.5 & 458 & 16 & 2.78 \\
4 & 440 & 15 & 2.83 \\
6 & 408 & 18 & 3.04 \\
\hline
\end{tabular}

Table 2 Peak emission wavelengths, lattice parameters, FWHM, and band gaps with ' $x$ ' in $\operatorname{MAPb}\left(\left.\mathrm{Br}_{x}\right|_{1-x}\right)_{3}$ at a fixed amount of ligand (1L)

\begin{tabular}{lllll}
\hline & $\begin{array}{l}\text { Peak } \\
\text { emission } \\
\text { wavelength } \\
(\mathrm{nm})\end{array}$ & $\begin{array}{l}\text { Lattice } \\
\text { parameter } \\
(\AA)\end{array}$ & $\begin{array}{l}\text { FWHM } \\
(\mathrm{nm})\end{array}$ & $\begin{array}{l}\text { Band } \\
\text { gap (eV) }\end{array}$ \\
\hline 1 & 520 & 5.930 & 21 & 2.36 \\
0.9 & 528 & 5.942 & 24 & 2.26 \\
0.8 & 534 & 5.961 & 35 & 2.19 \\
0.7 & 562 & 6.004 & 35 & 2.18 \\
0.6 & 600 & 6.044 & 38 & 2.09 \\
0.5 & 630 & 6.076 & 41 & 2.00 \\
0 & 755 & 6.304 & 46 & 1.51 \\
\hline
\end{tabular}

unit cell of the nanocrystals. The main diffraction peaks of the nanocrystals in the cubic phase are at approximately $15^{\circ}$, and $30^{\circ}$, that correspond to the (100) and (200) planes, respectively. ${ }^{22}$ The sharp Bragg peaks reveal that these hybrid perovskites have high periodicity and good phase purity. ${ }^{57}$ Furthermore, the crystal phase of $\mathrm{MAPbBr}_{3}$ nanocrystals remains cubic for various ligand concentrations.

However, increasing the iodine content (decreasing ' $x$ ') at a fixed ligand concentration resulted in a shift of the Bragg peaks to smaller angles (see in magnified XRD patterns, Fig. 1a). The shift in the angles occurs when bromine ions are replaced by larger iodine ions. The increased ionic radius of iodine causes the lattice parameter to increase (Table 2). At high iodine ion concentration, the crystal structure becomes tetragonal. The tetragonal phase is defined by a pseudocubic lattice; $;^{58}$ therefore, the phase change remains a factor when considering how the amount of iodine present in the crystal structure could affect the lattice parameter. The pure $\mathrm{MAPbI}_{3}$ sample has two Bragg peaks at $28.15^{\circ}$ and $28.41^{\circ}$ (see in magnified XRD patterns, Fig. 1a), which correspond to (004) and (220) planes in the tetragonal $I 4 / \mathrm{mcm}$ phase, respectively. These peaks gradually split with increasing the iodine content, which is attributed to the decrease in crystal structure symmetry. The crystal structure changes from the cubic phase to the tetragonal phase at a particular ratio of bromine to iodine content by rotating the $\mathrm{PbX}_{6}$ octahedrons while maintaining corner sharing connectivity ${ }^{58}$ as depicted in Fig. $1 \mathrm{~b}$ and c (the primitive cells of the tetragonal structure of $\mathrm{MAPbI}_{3}$ and the cubic structure of $\mathrm{MAPbBr}_{3}$, respectively).

There are additional sharp peaks in XRD patterns at smaller angles $\left(<10^{\circ}\right)$ that indicate the presence of highly ordered superlattice-like arrangements of nanocrystals. ${ }^{59}$ Some of these large superstructures are due to nanoplatelets stacking face-toface and hence yielding low angle diffraction peaks. ${ }^{60} \mathrm{~A}$ simple analysis of these peaks gives a spacing of $\sim 1-4 \mathrm{~nm}$ that corresponds to the spacing between the nanocrystals.

The effects of the various ligand concentrations and halide compositions on the morphology of the nanocrystals were analyzed by using TEM images, which are shown in Fig. 2 . The images in Fig. 2a-e show $\mathrm{MAPbBr}_{3}$ nanocrystals with various ligand concentrations while the images in Fig. $2 \mathrm{f}-\mathrm{j}$ show $\operatorname{MAPb}\left(\mathrm{Br}_{x} \mathrm{I}_{1-x}\right)_{3}$ nanocrystals at a static ligand concentration. For $\mathrm{MAPbBr}_{3}$ nanocrystals, a high ligand concentration $(4 L)$ results in the formation of nanoplatelets of thickness $1.73 \mathrm{~nm}$ and width $15.6 \mathrm{~nm}$ (Fig. 2a), while decreasing the ligand concentration $(3.5 L$ and $3 L$ ) allows nanocrystals grow larger and faster, which resulted in thicker nanoplatelets of thickness $2.28 \mathrm{~nm}$ and width $19.08 \mathrm{~nm}$ (Fig. 2b). In Fig. 2c, the thickness increases slightly from that in Fig. $2 \mathrm{~b}$ to $2.34 \mathrm{~nm}$ and
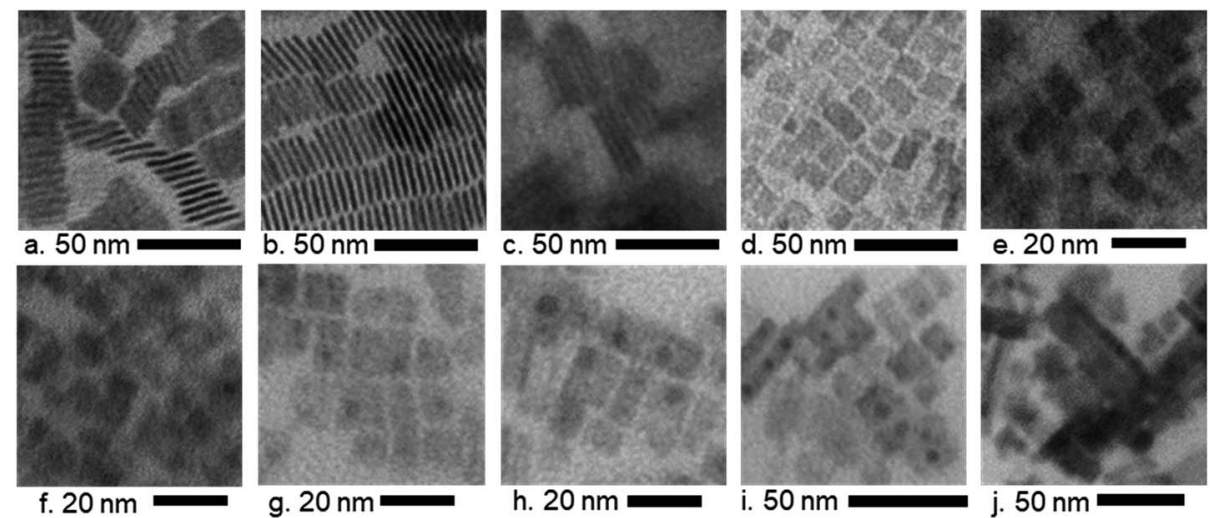

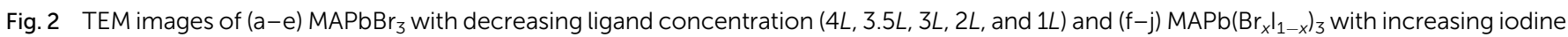
content $(x=0.8, x=0.6, x=0.5, x=0.4$, and $x=0)$ and fixed ligands (1L). 

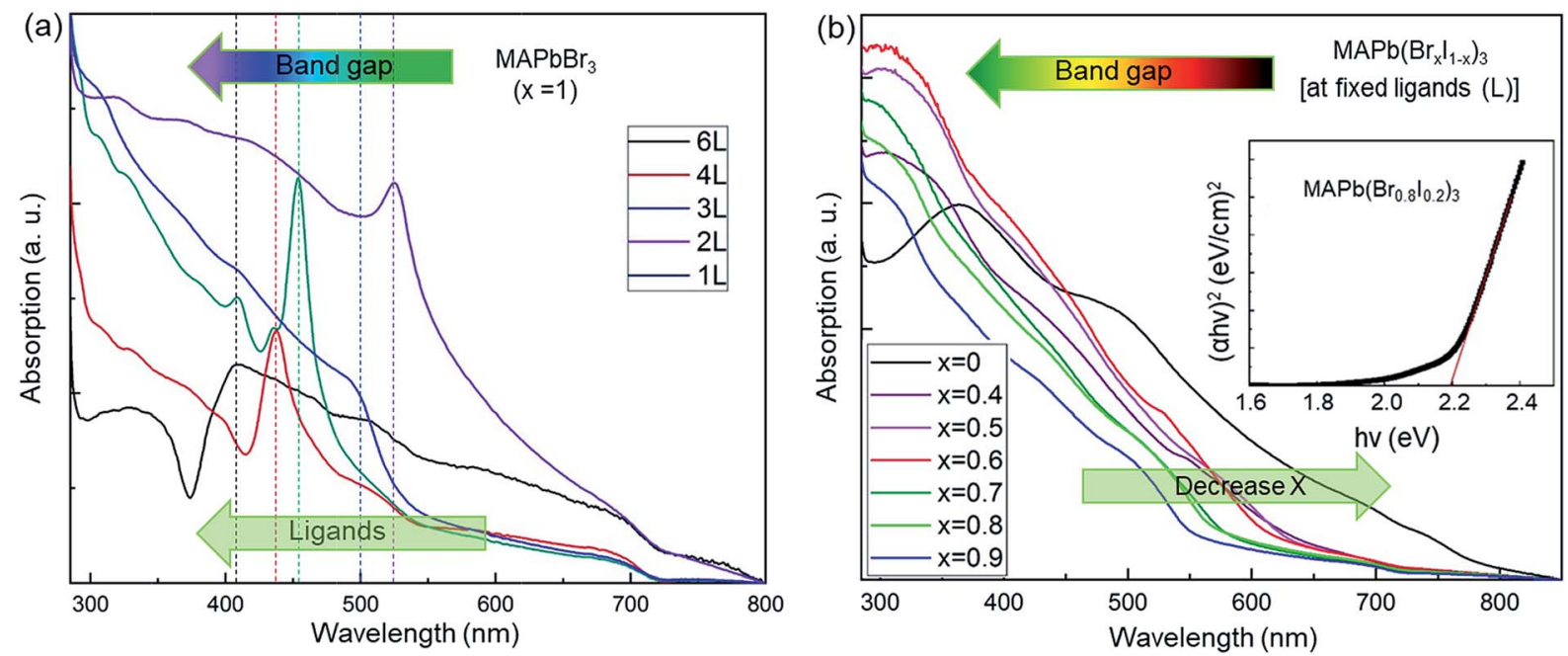

Fig. 3 Optical absorption spectra of (a) $\mathrm{MAPbBr}_{3}$ with ligand concentration variation and (b) $\mathrm{MAPb}\left(\mathrm{Br}_{x} \mathrm{l}_{1-x}\right)_{3}$ with 'Br' and 'l' composition variation at a fixed ligand amount (1L), and inset is the Tauc plot of the sample at $x=0.8$.

the width increases substantially to $25.2 \mathrm{~nm}$. The shape and orientation of these nanocrystals are already documented in the literature as face-to-face stacking nanoplatelets. ${ }^{61,62}$ The highly ordered growth in the $4 L$ and $3.5 L$ samples (average spacing of nanoplatelets $1.83 \mathrm{~nm}$ and $1.30 \mathrm{~nm}$, respectively, Fig. $2 a$ and $b$ ) that has been found in the TEM images is in agreement with the very strong low-angle superstructure peaks observed in the XRD patterns of these samples. However, further decreasing the ligand concentration $(2 L)$ causes a transformation in the structure of the nanocrystals from nanoplatelets into other rectangular nanocrystals with a side length of $6.3 \mathrm{~nm}$ (Fig. 2d). With a fixed ligand concentration $(1 L)$, increasing the iodine content in bromide-based perovskites increases the size of the formed nanocrystals from average side lengths $6.8 \mathrm{~nm}$ (Fig. 2e) to $8.7 \mathrm{~nm}$ (Fig. 2f), $10.9 \mathrm{~nm}$ (Fig. 2g), $13.9 \mathrm{~nm}$ (Fig. 2h), $19.7 \mathrm{~nm}$ (Fig. 2i), and $19.9 \mathrm{~nm}$ (Fig. 2j). The morphology remains unchanged for the mixture of 'Br' and 'I'-based nanocrystals. Nevertheless, these rectangular nanocrystals are comprised of sets of nanoplatelets, which self-assemble into larger structures. ${ }^{22}$ The periodic and well-ordered patterns in the TEM images also exhibit the existence of superlattice-like structures indicated by low-angle diffraction peaks in Fig. 1.

UV-visible absorption spectra (Fig. 3) were taken to study the effect of various ligand concentrations and halide compositions on the band gap of the nanocrystals. The absorption spectra of $\mathrm{MAPbBr}_{3}$ with different ligand concentrations are presented in Fig. 3a. We have approximated the band gap using the peak position of the absorption spectra. The absorption spectra of mixed-halide perovskites (' $\mathrm{Br}$ ' and ' $\mathrm{I}$ ') at a fixed ligand concentration are shown in Fig. $3 \mathrm{~b}$. These spectra indicate the strong absorption of the synthesized nanocrystals in the visible region. The value of the bandgaps was calculated using Tauc plot analyses which are shown in the inset of Fig. $3 b .^{63}$ The bandgap ranges from $3.04 \mathrm{eV}$ to $1.51 \mathrm{eV}$ and these values are summarized in Tables 1 and 2. The band gap tuning of $\mathrm{MAPbBr}_{3}$ was achieved through quantum confinement effects in the nanocrystals. The bandgap has a dependence on the ligand concentration while the nanocrystals remain smaller than the Bohr radius size limit because the quantum confinement effect is strongest with smaller nanocrystals and weakens as the size of the nanocrystals increases and disappears completely once the nanocrystals grow larger than the Bohr radius size limit. For mixed halide perovskites, however, the band edge varies via a changing ratio of smaller ' $\mathrm{Br}$ ' ions to larger 'I' ions in the crystal structure. It is known that the formation of the bandgap is a result of the bonding interaction between ' $\mathrm{Pb}$ ' and ' $\mathrm{Br} / \mathrm{I}$ ' of $\mathrm{ABX}_{3}$ perovskites. The bonding interaction between ' $\mathrm{Pb}$ ' and ' $\mathrm{Br}$ ' is stronger than that between ' $\mathrm{Pb}$ ' and ' $\mathrm{I} \cdot{ }^{64}$ Thus, there are larger bandgaps for the perovskites with a high bromine content than that of perovskites containing mostly or entirely iodine. The controllably tuned bandgap energy from violet to IR

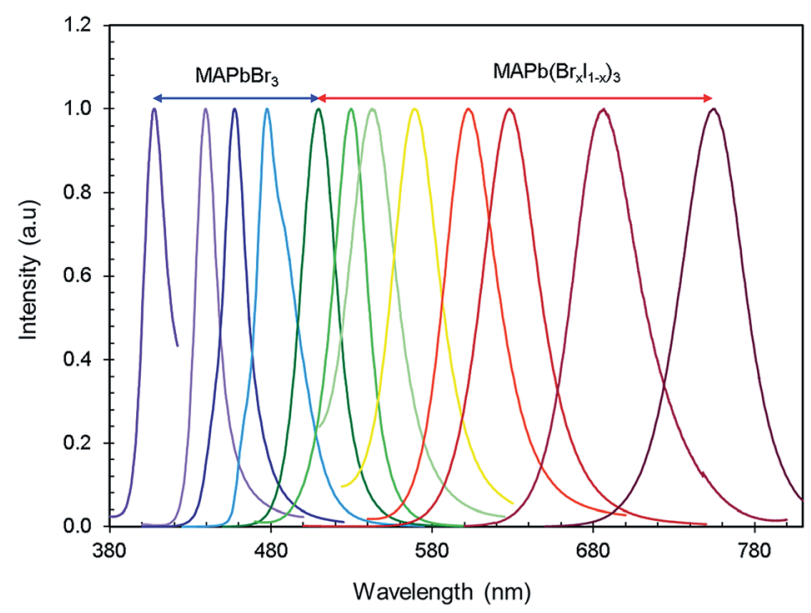

Fig. 4 The normalized PL emission spectra of halide perovskite nanocrystals over the range of 408-755 $\mathrm{nm}$ with varying ligand concentration for $\mathrm{MAPbBr}_{3}$ and composition of ' $\mathrm{Br}$ ' and ' $\mathrm{l}$ ' for $\operatorname{MAPb}\left(\left.\mathrm{Br}_{x}\right|_{1-x}\right)_{3}$. 

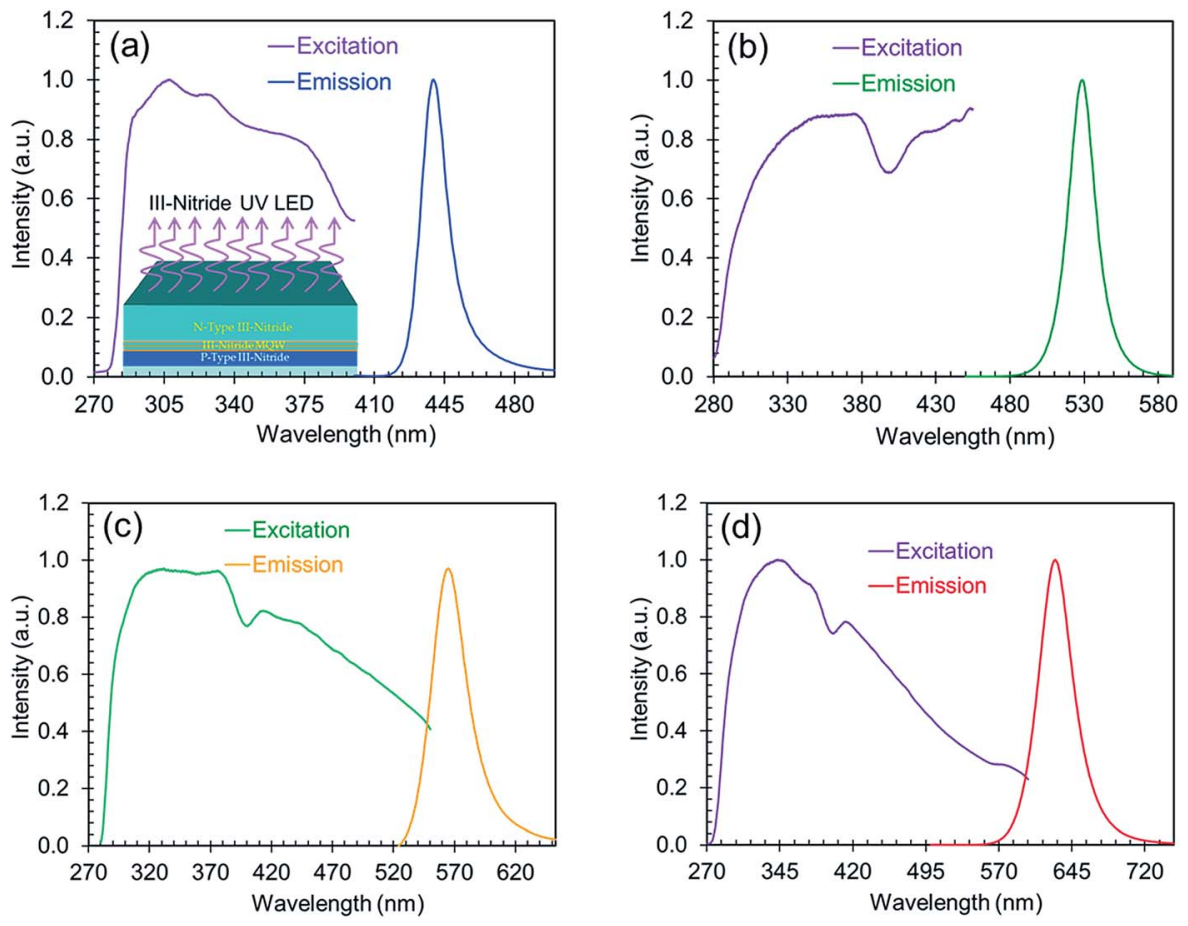

Fig. 5 The normalized excitation spectra along with the corresponding normalized emission (blue, green, yellow, and red) spectra.

by using only two types of halide ions leading to successful procurement of colorful optoelectronic devices.

Fig. 4 shows the PL emission of $\operatorname{MAPb}\left(\mathrm{Br}_{x} \mathrm{I}_{1-x}\right)_{3}$ perovskites synthesized at various ligand concentrations and different ' $\mathrm{Br}$ ' to 'I' ratios. Tunable emission was observed from $\mathrm{MAPbBr}_{3}$ nanocrystals by varying the ligand concentration with an excitation wavelength of $365 \mathrm{~nm}$. The peak position of the PL emission ranged from violet to green $(408-520 \mathrm{~nm})$. The ligand concentration affects the PL emission of the nanocrystals by restraining crystal growth as ligands bind to the outside of the nanocrystals during nucleation, thus making the structures smaller. This growth constraint gives rise to stronger quantum confinement effects, thus engineering the PL properties of the material. Then, the ratio of ' $\mathrm{Br}$ ' and ' $\mathrm{I}$ ' was adjusted for $\operatorname{MAPb}\left(\mathrm{Br}_{x} \mathrm{I}_{1-x}\right)_{3}$ at a fixed ligand concentration to obtain wavelength ranges from green to IR $(520-755 \mathrm{~nm})$ and these nanocrystals are excited at $450 \mathrm{~nm}$. The peak emission wavelengths are summarized in Tables 1 and 2. The PL emission spectra (violet-IR) are narrow and sharp as characterized by the fullwidth at half-maximum (FWHM). The range of values for the FWHM from blue to red is 15-41 nm (shown in Tables 1 and 2), which are comparatively smaller values than typically found for these compounds. ${ }^{34,65}$ The narrow FWHM implies high brightness, strong emission, and color purity in the PL emission of these perovskites which acts to increase the CRI of white light emission. ${ }^{6,67}$ Hence, we effectively tuned the PL from violet to IR using only two halides in the perovskite nanocrystals.

The FWHM of the PL emission in ligand tuned $\mathrm{MAPbBr}_{3}$ nanocrystals noticeably changes as the spectrum shifts to the red range. The FWHM reduction towards the blue and violet end of the spectrum is due to the effects that ligands have on crystal growth. The shorter crystal growth stage forces all nanocrystals to be smaller in size because there would not be enough time to create a significant size discrepancy between the growing nanocrystals during the shortened crystal growth stage during synthesis. As the ligand concentration decreases, the FWHM of PL emission increases. For mixed-halide perovskites, the increase in the FWHM as the iodine content increases is due to the inherently broader FWHM of $\mathrm{MAPbI}_{3}$ nanocrystals. ${ }^{68}$

The excitation spectra along with the corresponding emission spectra of the perovskites are shown in Fig. 5. These excitation spectra are broadband and extend from the UV to the visible range, which indicates the possibility of excitation by

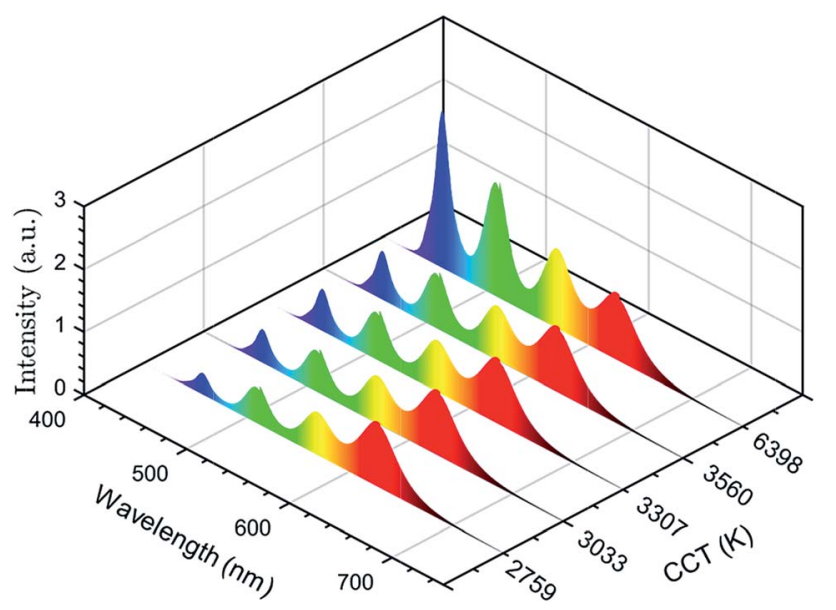

Fig. 6 Combination of the emission spectra from four different emissions for tuning the CCT of white light. 


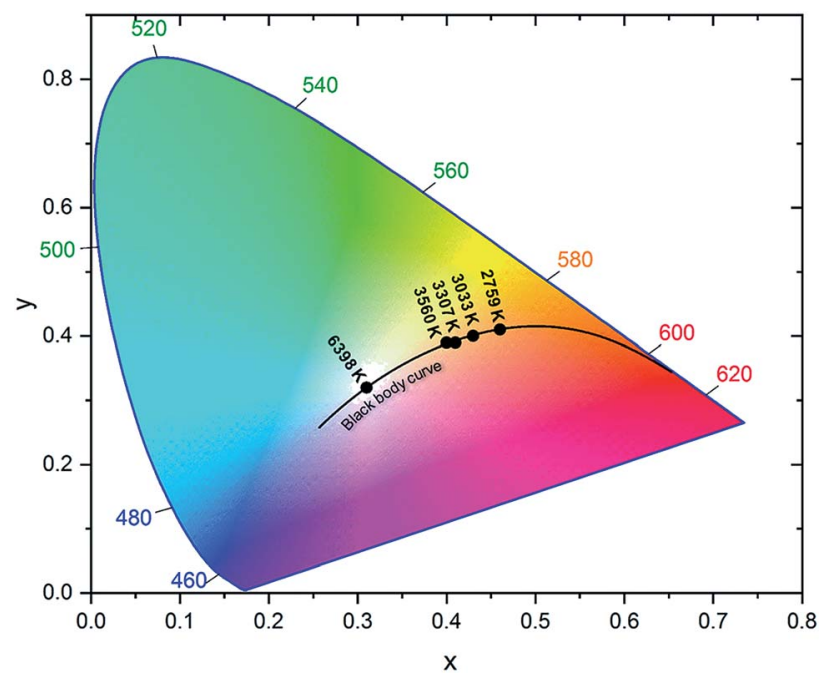

Fig. 7 CIE color coordinate diagram showing white light quality across the obtained range of CCT values.

a wide range of wavelengths. All the nanocrystals synthesized with varying ligand concentrations or anion ratios are therefore usable as down-conversion phosphors on a wide range of IIInitride LEDs for the development of white LED technology.

Furthermore, for the first time, we have demonstrated a strategy to generate high-quality white light emission by combining nanocrystals of blue $(\lambda=458 \mathrm{~nm})$, green $(\lambda=510$ $\mathrm{nm})$, yellow $(\lambda=562 \mathrm{~nm})$, and red $(\lambda=630 \mathrm{~nm})$ emissions. Then, we estimated the CCT and CRI of white light obtained from these nanocrystals. The combined emission spectra, tuned for different CCTs, are presented in Fig. 6. The combination of the four emission spectra demonstrates that a relatively high integrated intensity of the yellow and red phosphors is suitable for warm white light whereas increasing the integrated intensity of blue and green phosphors will cool the light hue. The CIE color coordinate diagram of white light emission is shown in Fig. 7. The effect of tuning the CCT of white light is plotted on the black body curve of the CIE chart. The CIE coordinates corresponding to white light nearly match the Planckian locus, which reveals that the color temperature we obtained is suitable and comfortable for human eyes. Different CCT and CRI values with corresponding CIE coordinates are presented in Table 3. The maximum CRI value we obtained is 93.95 at a CCT of $2759 \mathrm{~K}$. To the best of our knowledge, the CRI value here is superior to previous reports for these materials. ${ }^{3,49}$ Higher CRI values with suitable CCTs can be obtained from the

Table 3 The values of CCT, CRI, CIE coordinates, and ratios between the integrated intensities of emission components

\begin{tabular}{llllllll}
\hline CCT $(\mathrm{K})$ & Blue & Green & Yellow & Red & CRI & $x$ & $y$ \\
\hline 2759 & 0.15 & 0.34 & 0.45 & 1 & 93.95 & 0.46 & 0.41 \\
3033 & 0.25 & 0.38 & 0.50 & 1 & 93.71 & 0.43 & 0.40 \\
3307 & 0.31 & 0.45 & 0.54 & 1 & 93.53 & 0.41 & 0.39 \\
3560 & 0.36 & 0.52 & 0.58 & 1 & 93.33 & 0.40 & 0.39 \\
6398 & 1.17 & 1.07 & 0.85 & 1 & 90.59 & 0.31 & 0.33
\end{tabular}

combination of nanocrystals with different emissions. In addition, one of the CIE coordinates obtained is $(0.31,0.33)$ which is very close to the standard neutral white light $(0.33,0.33)$. These results indicate that perovskite nanocrystals $\operatorname{MAPb}\left(\mathrm{Br}_{x} \mathrm{I}_{1-x}\right)_{3}$ have the potential to replace the conventional phosphors for next-generation light emitting devices.

\section{Conclusion}

In summary, nanocrystals with PL emission spectra ranging from violet to IR (408-755 nm) have been synthesized by adjusting the ligand concentration in $\mathrm{MAPbBr}_{3}$ and anion ratios of ' $\mathrm{Br}$ ' to ' $\mathrm{I}$ ' in $\operatorname{MAPb}\left(\mathrm{Br}_{x} \mathrm{I}_{1-x}\right)_{3}$ while keeping the ligand concentration constant. The bandgap is controllably tuned through the range of 3.04-1.51 eV. Thus, optoelectronic devices with a broad color gamut are now obtainable using two halide types rather than the traditional three, including $\mathrm{MAPbCl}_{3}$ perovskites. In addition, we have demonstrated, as a first for this class of materials, a CCT estimation extending from cool to warm white emission with CRI values of up to 93.95. To our knowledge, a CRI of 93.95 is the highest value achieved using these materials. This exceptional light quality demonstrates the potential use of this material as the next generation of photon down-conversion phosphors for lighting devices.

\section{Conflicts of interest}

There are no conflicts to declare.

\section{Acknowledgements}

The authors acknowledge the financial support by The University of Tulsa through a Start-up Fund. The authors thank Dr Parameswar Harikumar's Semiconductor Characterization Laboratory for allowing the use of equipment for absorption measurements.

\section{Notes and references}

1 M. G. Kibria and Z. Mi, J. Mater. Chem. A, 2016, 4, 2801-2820. 2 N. Guan, X. Dai, A. Messanvi, H. Zhang, J. Yan, E. Gautier, C. Bougerol, F. H. Julien, C. Durand, J. Eymery and M. Tchernycheva, ACS Photonics, 2016, 3, 597-603.

3 J. McKittrick and L. E. Shea-Rohwer, J. Am. Ceram. Soc., 2014, 97, 1327-1352.

4 N. Xu Lin, N. Thi Ngoc Nhien, C. Vinh Thang and D. Mau Chien, Adv. Nat. Sci.: Nanosci. Nanotechnol., 2010, 1, 025015. 5 H. Z. Hai Guo, R. F. Wei, M. D. Zheng and LiH. Zhang, Opt. Express, 2011, 19, A201-A206.

6 P. Zhu, H. Zhu, W. Qin, B. H. Dantas, W. Sun, C.-K. Tan and N. Tansu, J. Appl. Phys., 2016, 119, 124305.

7 P. F. Smet, K. Korthout, J. E. Van Haecke and D. Poelman, Mater. Sci. Eng., B, 2008, 146, 264-268.

8 W. Wang and P. Zhu, Opt. Express, 2018, 26, 34820-34829.

9 P. Zhu, W. Wang, H. Zhu, P. Vargas and A. Bont, IEEE Photonics J., 2018, 10, 4500210.

10 H. Cui, P.-F. Zhu, H.-Y. Zhu, H.-D. Li and Q.-L. Cui, Chin. Phys. B, 2014, 23, 057801. 
11 T. Guner and M. M. Demir, Phys. Status Solidi A, 2018, 215, 1800120.

12 M. M. Lee, J. Teuscher, T. Miyasaka, T. N. Murakami and H. J. Snaith, Science, 2012, 338, 643-647.

13 L. Li, Y. Chen, Z. Liu, Q. Chen, X. Wang and H. Zhou, Adv. Mater., 2016, 28, 9862-9868.

14 L. Zhang, Q. Zeng and K. Wang, J. Phys. Chem. Lett., 2017, 8, 3752-3758.

15 R. Naphade, S. Nagane, G. S. Shanker, R. Fernandes, D. Kothari, Y. Zhou, N. P. Padture and S. Ogale, ACS Appl. Mat. Interfaces, 2016, 8, 854-861.

16 S. Gonzalez-Carrero, R. E. Galian and J. Pérez-Prieto, Opt. Express, 2016, 24, A285-A301.

17 Z. Fan, K. Sun and J. Wang, J. Mater. Chem. A, 2015, 3, 1880918828.

18 V. A. Hintermayr, A. F. Richter, F. Ehrat, M. Döblinger, W. Vanderlinden, J. A. Sichert, Y. Tong, L. Polavarapu, J. Feldmann and A. S. Urban, Adv. Mater., 2016, 28, 94789485.

19 G. C. Adhikari, H. Zhu, P. A. Vargas and P. Zhu, J. Phys. Chem. C, 2018, 122, 15041-15046.

20 G. C. Adhikari, P. A. Vargas, H. Zhu and P. Zhu, J. Phys. Chem. $C, 2019,123,1406-1412$.

21 S. Gonzalez-Carrero, G. M. Espallargas, R. E. Galian and J. Perez-Prieto, J. Mater. Chem. A, 2015, 3, 14039-14045.

22 Y. Ling, Z. Yuan, Y. Tian, X. Wang, J. C. Wang, Y. Xin, K. Hanson, B. Ma and H. Gao, Adv. Mater., 2016, 28, 305-311.

23 Q. Pan, H. Hu, Y. Zou, M. Chen, L. Wu, D. Yang, X. Yuan, J. Fan, B. Sun and Q. Zhang, J. Mater. Chem. C, 2017, 5, 10947-10954.

24 F. Zhang, H. Zhong, C. Chen, X.-g. Wu, X. Hu, H. Huang, J. Han, B. Zou and Y. Dong, ACS Nano, 2015, 9, 4533-4542.

25 I. Levchuk, A. Osvet, X. Tang, M. Brandl, J. D. Perea, F. Hoegl, G. J. Matt, R. Hock, M. Batentschuk and C. J. Brabec, Nano Lett., 2017, 17, 2765-2770.

26 S. A. Veldhuis, P. P. Boix, N. Yantara, M. Li, T. C. Sum, N. Mathews and S. G. Mhaisalkar, Adv. Mater., 2016, 28, 6804-6834.

27 D. B. Khadka, Y. Shirai, M. Yanagida, T. Noda and K. Miyano, ACS Appl. Mater. Interfaces, 2018, 10, 22074-22082.

28 R. Naphade, B. Zhao, J. M. Richter, E. Booker, S. Krishnamurthy, R. H. Friend, A. Sadhanala and S. Ogale, ACS Appl. Mater. Interfaces, 2017, 4, 1700562.

29 E. L. Unger, L. Kegelmann, K. Suchan, D. Sorell, L. Korte and S. Albrecht, J. Mater. Chem. A, 2017, 5, 11401-11409.

30 S. A. Kulkarni, T. Baikie, P. P. Boix, N. Yantara, N. Mathews and S. Mhaisalkar, J. Mater. Chem. A, 2014, 2, 9221-9225.

31 B. J. Bohn, Y. Tong, M. Gramlich, M. L. Lai, M. Döblinger, K. Wang, R. L. Z. Hoye, P. Müller-Buschbaum, S. D. Stranks, A. S. Urban, L. Polavarapu and J. Feldmann, Nano Lett., 2018, 18, 5231-5238.

32 L. Zhang, C. Liu, L. Wang, C. Liu, K. Wang and B. Zou, Angew. Chem., Int. Ed., 2018, 57, 11213-11217.

33 Y. Tong, M. Fu, E. Bladt, H. Huang, A. F. Richter, K. Wang, P. Müller-Buschbaum, S. Bals, P. Tamarat, B. Lounis, J. Feldmann and L. Polavarapu, Angew. Chem., Int. Ed., 2018, 57, 16094-16098.
34 J. Xing, F. Yan, Y. Zhao, S. Chen, H. Yu, Q. Zhang, R. Zeng, H. V. Demir, X. Sun, A. Huan and Q. Xiong, ACS Nano, 2016, 10, 6623-6630.

35 Z.-K. Tan, R. S. Moghaddam, M. L. Lai, P. Docampo, R. Higler, F. Deschler, M. Price, A. Sadhanala, L. M. Pazos, D. Credgington, F. Hanusch, T. Bein, H. J. Snaith and R. H. Friend, Nat. Nanotechnol., 2014, 9, 687-692.

36 X. Qin, H. Dong and W. Hu, Sci. China Mater., 2015, 58, 186191.

37 H. Zhu, T. Cai, M. Que, J.-P. Song, B. M. Rubenstein, Z. Wang and O. Chen, J. Phys. Chem. Lett., 2018, 9, 4199-4205.

38 H. Shao, X. Bai, H. Cui, G. Pan, P. Jing, S. Qu, J. Zhu, Y. Zhai, B. Dong and H. Song, Nanoscale, 2018, 10, 1023-1029.

39 T. Li, Y. Pan, Z. Wang, Y. Xia, Y. Chen and W. Huang, J. Mater. Chem. A, 2017, 5, 12602-12652.

40 D. B. Khadka, Y. Shirai, M. Yanagida and K. Miyano, J. Mater. Chem. C, 2018, 6, 162-170.

41 S. Pont, D. Bryant, C.-T. Lin, N. Aristidou, S. Wheeler, X. Ma, R. Godin, S. A. Haque and J. R. Durrant, J. Mater. Chem. A, 2017, 5, 9553-9560.

42 A. Polman, M. Knight, E. C. Garnett, B. Ehrler and W. C. Sinke, Science, 2016, 352, 4424.

43 P. Wangyang, H. Sun, X. Zhu, D. Yang, X. Gao, W. Liu, Y. Chen, H. Tian and S. Huanglong, Phys. Status Solidi A, 2017, 214, 1700538.

44 L. Li, Z. Sun, P. Wang, W. Hu, S. Wang, C. Ji, M. Hong and J. Luo, Angew. Chem., Int. Ed., 2017, 56, 12150-12154.

45 J. Zhang, X. Yang, H. Deng, K. Qiao, U. Farooq, M. Ishaq, F. Yi, H. Liu, J. Tang and H. Song, Nano-Micro Lett., 2017, 9, 36.

46 Z. Gu, K. Wang, W. Sun, J. Li, S. Liu, Q. Song and S. Xiao, Adv. Opt. Mater., 2016, 4, 472-479.

47 Y. En-Ping, Y. Zhanlue, M. Lei, S. Pengyu, D. Shiqi, Y. Ye and Y. Yang, Adv. Mater., 2017, 29, 1606859.

48 K. T. Shimizu, M. Böhmer, D. Estrada, S. Gangwal, S. Grabowski, H. Bechtel, E. Kang, K. J. Vampola, D. Chamberlin, O. B. Shchekin and J. Bhardwaj, Photonics Res., 2017, 5, A1-A6.

49 S. Pathak, N. Sakai, F. Wisnivesky Rocca Rivarola, S. D. Stranks, J. Liu, G. E. Eperon, C. Ducati, K. Wojciechowski, J. T. Griffiths, A. A. Haghighirad, A. Pellaroque, R. H. Friend and H. J. Snaith, Chem. Mater., 2015, 27, 8066-8075.

50 Y.-W. Zhang, G. Wu, H. Dang, K. Ma and S. Chen, Ind. Eng. Chem. Res., 2017, 56, 10053-10059.

51 E. R. Dohner, A. Jaffe, L. R. Bradshaw and H. I. Karunadasa, J. Am. Chem. Soc., 2014, 136, 13154-13157.

52 L. Mao, Y. Wu, C. C. Stoumpos, M. R. Wasielewski and M. G. Kanatzidis, J. Am. Chem. Soc., 2017, 139, 5210-5215.

53 Z. Zhuang, C. Peng, G. Zhang, H. Yang, J. Yin and H. Fei, Angew. Chem., Int. Ed., 2017, 56, 14411-14416.

54 C. Peng, Z. Zhuang, H. Yang, G. Zhang and H. Fei, Chem. Sci., 2018, 9, 1627-1633.

55 R. K. Misra, L. Ciammaruchi, S. Aharon, D. Mogilyansky, L. Etgar, I. Visoly-Fisher and E. A. Katz, ChemSusChem, 2016, 9, 2572-2577. 
56 T. Baikie, Y. Fang, J. M. Kadro, M. Schreyer, F. Wei, S. G. Mhaisalkar, M. Graetzel and T. J. White, J. Mater. Chem. A, 2013, 1, 5628-5641.

57 L.-d. Tang, H. Mei, B. Wang and S. Peng, J. Mater. Sci.: Mater. Electron., 2015, 26, 8726-8731.

58 J. H. Noh, S. H. Im, J. H. Heo, T. N. Mandal and S. I. Seok, Nano Lett., 2013, 13, 1764-1769.

59 Y. Nagaoka, K. Hills-Kimball, R. Tan, R. Li, Z. Wang and O. Chen, Adv. Mater., 2017, 29, 1606666.

60 Y. Nagaoka, R. Tan, R. Li, H. Zhu, D. Eggert, Y. A. Wu, Y. Liu, Z. Wang and O. Chen, Nature, 2018, 561, 378-382.

61 Q. A. Akkerman, S. G. Motti, A. R. Srimath Kandada, E. Mosconi, V. D'Innocenzo, G. Bertoni, S. Marras, B. A. Kamino, L. Miranda, F. De Angelis, A. Petrozza, M. Prato and L. Manna, J. Am. Chem. Soc., 2016, 138, 1010-1016.

62 O. Vybornyi, S. Yakunin and M. V. Kovalenko, Nanoscale, 2016, 8, 6278-6283.
63 B. P. Kafle, S. Acharya, S. Thapa and S. Poudel, Ceram. Int., 2016, 42, 1133-1139.

64 Y. Heng-Yun, L. Wei-Qiang, H. Chun-Li, Z. Yi, Y. Yu-Meng, M. Jiang-Gao, L. Peng-Fei and X. Ren-Gen, Adv. Mater., 2016, 28, 2579-2586.

65 G. Li, F. W. R. Rivarola, N. J. L. K. Davis, S. Bai, T. C. Jellicoe, F. de la Peña, S. Hou, C. Ducati, F. Gao, R. H. Friend, N. C. Greenham and Z.-K. Tan, Adv. Mater., 2016, 28, 35283534.

66 G. Li, Z.-K. Tan, D. Di, M. L. Lai, L. Jiang, J. H.-W. Lim, R. H. Friend and N. C. Greenham, Nano Lett., 2015, 15, 2640-2644.

67 S. Zhuang, X. Ma, D. Hu, X. Dong and B. Zhang, Ceram. Int., 2018, 44, 4685-4688.

68 D. Li, G. Wang, H.-C. Cheng, C.-Y. Chen, H. Wu, Y. Liu, Y. Huang and X. Duan, Nat. Commun., 2016, 7, 11330. 